\title{
CALCEOLARIA DICHOTOMA SUBSP. COLIMANA (CALCEOLARIACEAE), UNA SUBESPECIE NUEVA DEL OCCIDENTE DE MÉXICO
}

\author{
Eloy Padilla-Velarde y Ramón Cuevas-Guzmán \\ Universidad de Guadalajara, Centro Universitario de la Costa Sur, Instituto \\ Manantlán de Ecología y Conservación de la Biodiversidad, Avenida \\ Independencia Nacional 151, 48900 Autlán de Navarro, Jalisco, México. \\ rcuevas@cucsur.udg.mx
}

\section{RESUMEN}

Se describe e ilustra a Calceolaria dichotoma subsp. colimana, una subespecie nueva del estado de Colima en el occidente de México. C. dichotoma pertenece al subgénero Cheiloncos y sección Micranthera, con anterioridad sólo registrados de los Andes, desde Colombia hasta el norte de Argentina. El hallazgo del taxon en Colima incrementa en miles de kilómetros al norte la distribución conocida del subgénero, la sección y la especie.

Palabras clave: Calceolaria, Cheiloncos, Colima, México, Micranthera.

\begin{abstract}
Calceolaria dichotoma subsp. colimana is described and illustrated as a new subspecies from the state of Colima in western Mexico. C. dichotoma belongs to subgenus Cheiloncos and section Micranthera, previously only known from the Andes, from Colombia to the north of Argentina. The finding of the taxon in Colima increases thousands of kilometers to the north the known distribution of the subgenus, section and species.

Key words: Calceolaria, Cheiloncos, Colima, Mexico, Micranthera.

En una de las exploraciones para conocer y registrar plantas vasculares que crecen silvestres en la parte alta del arroyo de Agua Fría, municipio de Minatitlán, Colima, se encontró una especie de Calceolaria. Después de revisar la literatura
\end{abstract}


pertinente para el género y ejemplares de los herbarios CHAPA, IBUG, MEXU y ZEA, se identificó inicialmente como Calceolaria dichotoma Lamarck, pero dada su ubicación geográfica, así como sus características morfológicas, no del todo coincidentes, se le propone como una subespecie de este último taxon.

Calceolaria dichotoma Lamarck subsp. colimana Padilla-V. \& Cuevas subsp. nov. Figs. 1, 2.

Herba annua 8-30 cm alta; folia decussata, lamina 11-25 mm longa, 6-18 mm lata, petiolo 1-5 mm longo; flores axillares, 1 in axilla, 2 per nodum; pedicelli 3-11 $\mathrm{mm}$ longi; calyx 4-sectus, segmentis inaequalibus, ciliatis, $1.7-2.5 \mathrm{~mm}$ longis, $1.1-2$ $\mathrm{mm}$ latis; corolla lutea vel luteo-aurantiaca, sine maculis, labio superiore curvato, $1.5-2 \times 2-2.5 \mathrm{~mm}$, labio inferiore sacciforme, 3.5-5.5 x 3.2-5 mm; elaeophorus adest; stamina filamentis laminatis $1.5-2 \mathrm{~mm}$ longis, antherae $0.5-0.75 \mathrm{~mm}$ longae, 0.4-0.5 mm latae, thecae aequales, divaricatae vel leviter deflexae, totae dehiscentes; capsula turbinata 4.3-7 x 3-3.9 mm; semina ellipsoidea $0.4-0.5 \times 0.2-0.3 \mathrm{~mm}$, glabra.

Hierba erecta anual, 8-30 cm de altura, con entrenudos de 1-4 $\mathrm{cm}$ de largo; la mayor parte de la planta cubierta con pelos glandulares, septados. Hojas simples, decusadas; láminas anchamente ovadas a rómbicas, 11-25 $\mathrm{mm}$ de largo, 6-18 mm de ancho, ápice agudo, margen subentero a serrado, base anchamente cuneada a subcordada, a veces oblicua, haz verde claro con pelos septados, en su mayoría no glandulares, 0.4-1 mm de longitud, envés verde blanquecino con pelos más cortos y dispersos que en el haz, glandulares, nerviación craspedódroma, con (2)3 o 4(5) pares de nervios secundarios, algunos de los nervios terciarios formando los dientes, los cuales con frecuencia son glandulares; pecíolos 1-5 $\mathrm{mm}$ de longitud. Inflorescencia difusa, ocupando la mayor parte del cuerpo aéreo de la planta, flores axilares, una por axila, dos por nudo, ambas con la misma orientación, en delgados pedicelos de 3-11 mm de largo, uno de ellos divaricado respecto al eje del tallo al convertirse en fruto y el otro más o menos paralelo al entrenudo superior. Cáliz de cuatro sépalos desiguales, ciliados, ovados, anchamente ovados o en ocasiones elípticos, 1.7-2.5 mm de largo, 1.1-2 mm de ancho en la antesis, acrescentes en el fruto y entonces de $4.7 \mathrm{~mm}$ de largo, $3.6 \mathrm{~mm}$ de ancho. Corola tipo X (Molau, 1988), de color amarillo claro a amarillo anaranjado, sin ninguna banda ni puntos coloridos en alguna parte, labio superior arqueado, 1.5-2 x 2-2.5 mm, finalizando en un ápice que se trunca y mide $\pm 0.3 \mathrm{~mm}$ de ancho en su parte terminal; labio inferior, 3.5-5.5 
Padilla-Velarde y Cuevas-Guzmán: Calceolaria dichotoma subsp. colimana, subespecie nueva de México
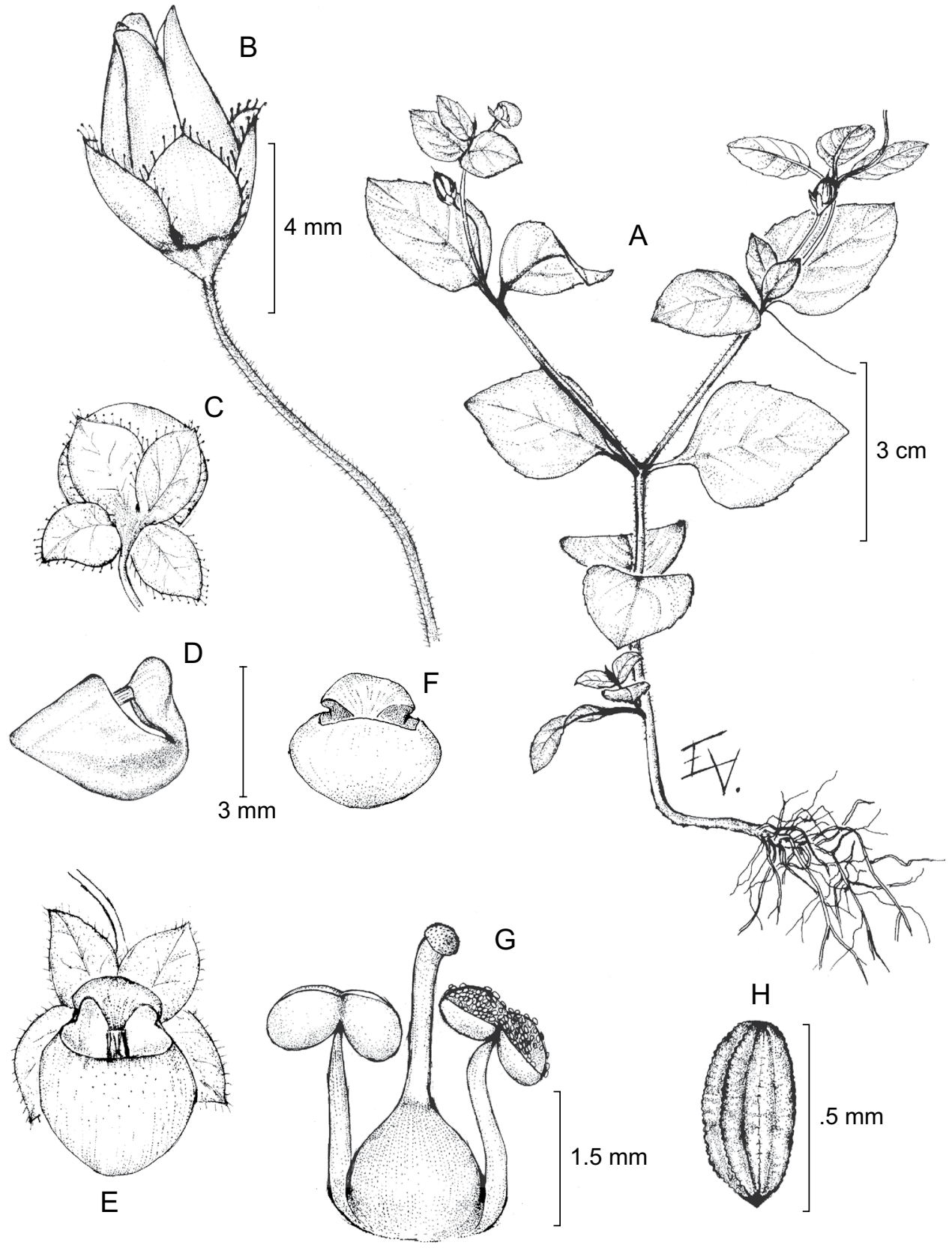

Fig. 1. Calceolaria dichotoma subsp. colimana. A. planta completa; B. detalle del fruto; C. vista dorsal de la flor, mostrando los sépalos; D. vista lateral de la flor; E. vista superior de la flor; F. vista frontal de la flor; G. pistilo y androceo; H. semilla (Padilla-V. 1500). 
x 3.2-5 mm, sacciforme en 40-60\% de su longitud; elaióforo presente. Estambres 2, glabros; filamentos laminares y más ensanchados cerca de su ápice, $1.5-2 \mathrm{~mm}$ de longitud; anteras, 0.5-0.75 $\mathrm{mm}$ de largo, 0.4-0.5 mm de ancho, tecas divaricadas a reclinadas, amarillas, abren completamente, tornándose planas y blancas. Ovario glandular pubescente; estilo, 1.1-1.3 mm, decurvado en el ápice; estigma capitado. Cápsula cónica, 4.3-7 × 3-3.9 mm, esparcidamente glandular pilosa a casi glabra, de color castaño claro, algo translúcida, abriendo por 4 valvas; conteniendo \pm 90 semillas por fruto; pedicelos, $14-19 \mathrm{~mm}$ de largo. Semillas elipsoides, 0.4-0.5 mm de largo, $0.2-0.3 \mathrm{~mm}$ de ancho, con 6-8 costillas longitudinales y \pm 15 transversales, glabras, de color castaño-rojizo.

Tipo: México, Colima: La Gloria Escondida, ejido Agua Fría, municipio de Minatitlán, bosque mesófilo de montaña perturbado, $19^{\circ} 18^{\prime} \mathrm{N}$ y $104^{\circ} 01^{\prime} \mathrm{O}, 1650 \mathrm{~m}$ s.n.m. 12/09/2007, Padilla-V. 1500, holotipo en ZEA.

Paratipos: México, Colima: La Gloria Escondida, ejido Agua Fría, municipio de Minatitlán, bosque mesófilo de montaña perturbado, 1600 m s.n.m. 14/09/2004, Padilla-V. 1354 (ZEA).

Se le conoce sólo de la localidad tipo. Crece en áreas abiertas cerca de los cauces de arroyos, en bosque mesófilo de montaña, donde convive con Achimenes antirrhina (DC.) C.V. Morton, Alternanthera lanceolata (Benth.) Schinz, Carpinus tropicalis (J.D. Sm.) Lundell, Drymaria cordata (L.) Willd. ex Roem. \& Schult., Oplismenus compositus (L.) P. Beauv., Oxalis alpina (Rose) R. Knuth, Ranunculus petiolaris Kunth ex DC., Scutellaria purpurascens Sw. y Senna foetidissima (G. Don) H.S. Irwin \& Barneby, entre otras.

Florece y fructifica en agosto y septiembre.

El nombre hace alusión al estado de Colima en el occidente de México, donde la subespecie fue encontrada.

Por sus filamentos dos veces más largos que las anteras, C. dichotoma se ha ubicado en el subgénero Cheilonchos. Por tratarse de hierbas delicadas y anuales, con hojas pecioladas, láminas ovadas y las inflorescencias cubriendo la mayor parte aérea, tiene acomodo en la sección Micranthera (Molau, 1981; Molau, 1988). C. dichotoma subsp. colimana se distingue de la subespecie típica en sus estructuras 
Padilla-Velarde y Cuevas-Guzmán: Calceolaria dichotoma subsp. colimana, subespecie nueva de México
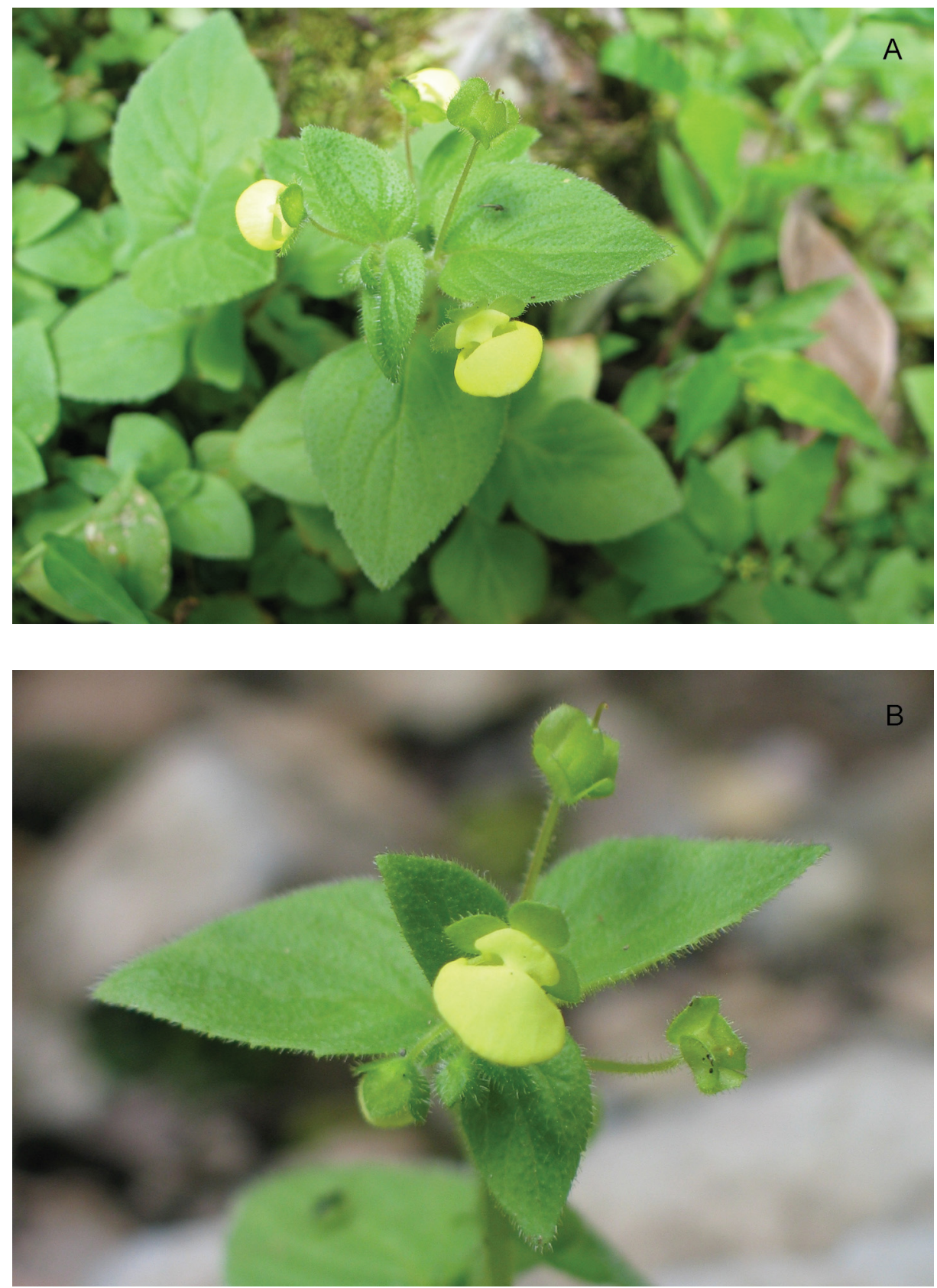

Fig. 2. A. Hábito y vista superior de las flores de C. dichotoma subsp. colimana, así como la disposición y forma de las hojas; B. Acercamiento de hojas, flores y frutos. 
florales, en general más pequeñas, en la carencia de bandas o puntos coloridos en la garganta y en sus filamentos laminares. Otras diferencias entre las dos subespecies se presentan en el Cuadro 1.

Cuadro 1. Comparación de los principales caracteres de diferenciación entre Calceolaria dichotoma subsp. dichotoma y C. dichotoma subsp. colimana.

\begin{tabular}{|c|c|c|}
\hline Caracter & C. dichotoma subsp. dichotoma & C. dichotoma subsp. colimana \\
\hline Tamaño de la planta & $5-50 \mathrm{~cm}$ de altura & $8-30 \mathrm{~cm}$ de altura \\
\hline Cáliz en la antesis & $1.7-4 \times 1.8-3.6 \mathrm{~mm}$ & $1.7-2.5 \times 1.1-2 \mathrm{~mm}$ \\
\hline Garganta de la corola & $\begin{array}{l}\text { con una banda transversal o dos } \\
\text { a muchos puntos rojizos }\end{array}$ & $\begin{array}{l}\text { sin banda transversal, ni puntos } \\
\text { coloridos }\end{array}$ \\
\hline $\begin{array}{l}\text { Labio superior de la } \\
\text { corola }\end{array}$ & $\begin{array}{l}2-4 \times 3-5 \mathrm{~mm} \text {, finalizando en } \\
\text { un ápice que se trunca y mide } \\
\text { de } 1-2 \mathrm{~mm} \text { de ancho en su parte } \\
\text { terminal }\end{array}$ & $\begin{array}{l}1.5-2 \times 2-2.5 \mathrm{~mm} \text {, finalizando } \\
\text { en un ápice que se trunca y } \\
\text { mide } \pm 0.3 \mathrm{~mm} \text { de ancho en } \mathrm{su} \\
\text { parte terminal }\end{array}$ \\
\hline $\begin{array}{l}\text { Labio inferior de la } \\
\text { corola }\end{array}$ & $4-9 \times 4-8 \mathrm{~mm}$ & $3.5-5.5 \times 3.2-5 \mathrm{~mm}$ \\
\hline Filamentos & filiformes, $1.4-2.5 \mathrm{~mm}$ de largo & laminares, $1.5-2 \mathrm{~mm}$ de largo \\
\hline Estilo & $1.2-2 \mathrm{~mm}$ de longitud & 1.1-1.3 mm de longitud \\
\hline Distribución & $\begin{array}{l}\text { norte de Colombia a Perú y las } \\
\text { Islas Galápagos }\end{array}$ & Colima, México \\
\hline Altitud & $70-3700 \mathrm{~m}$ & $1600-1650 \mathrm{~m}$ \\
\hline
\end{tabular}

Calceolaria es un género de distribución principalmente neotropical, cuyo centro de origen y diversificación se encuentra en los Andes, en la región denominada por Molau (1988) como la División Latitudinal Media, una de las tres zonas latitudinales en que se dividió el área de distribución del género y que comprende los Andes, desde el centro de Perú hasta la parte media de Bolivia. Unas pocas especies penetran a Centroamérica y sólo cuatro llegan hasta México, registrando su límite septentrional de distribución en la Sierra Madre Occidental, en los estados de Jalisco y Durango (Méndez-Larios y Villaseñor, 2001). El subgénero Cheilonchos con sus secciones Rugosae y Micranthera, se distribuye principalmente en la División Latitudinal Sur, región que comprende el sur de los Andes, desde el centro de Bolivia hasta la Patagonia, en la Tierra del Fuego y las Islas Malvinas, y sólo C. dichotoma sobrepasa esta división, yendo hasta la parte central de Colombia y el sur de Perú (Molau, 1988). 
El hallazgo de C. dichotoma subsp. colimana en el occidente de México, plantea un interesante problema fitogeográfico, pues el hecho de que se le encuentre miles de kilómetros al norte de sus representantes conespecíficos del subgénero Cheilonchos y la sección Micranthera, podría ser explicado a través de dos hipótesis: 1) que la especie presentaba una distribución más amplia en el pasado y actualmente sólo representa una reliquia de esa distribución anterior más continua; 2) que se trate de una invasión relativamente reciente de la especie, la cual está sufriendo un proceso de diferenciación de las poblaciones originales. En general parece haber más elementos de apoyo a la segunda hipótesis, entre los cuales podrían señalarse: a) Calceolaria es un género cuyo origen es Sudamérica templada, razón por la cual la mayor diversidad se encuentra en los Andes (Molau, 1988), de donde parece haber iniciado el proceso de radiación hacia el norte, con una disminución en la riqueza de especies hacia la parte septentrional del continente; b) las especies que se presentan en Centroamérica y México, son casi siempre de amplia distribución, con sus principales centros de distribución en el norte de los Andes (Molau, 1988); y c) Calceolaria dichotoma es una planta con vasta tolerancia ecológica y con comportamiento malezoide, por lo que es de esperarse que tenga una gran plasticidad; por otro lado las semillas podrían ser transportadas a grandes distancias por viento, pues son muy pequeñas y livianas, y no es de descartarse que algún ciclón las haya llevado desde las Islas Galápagos hasta el occidente de México, lo cual también explicaría el porqué no se han encontrado poblaciones entre el estado de Colima y el norte de Colombia.

Muchas interrogantes tendrán que ser resueltas antes de contar con una respuesta satisfactoria, entre otras ¿de dónde, cómo y cuándo llegó C. dichotoma al occidente de México? Estudios moleculares, citológicos, anatómicos y fisiológicos serán fundamentales para precisar el origen y las relaciones de la nueva subespecie propuesta.

\section{AGRADECIMIENTOS}

Agradecemos a los pobladores del ejido Agua Fría, por permitirnos la exploración y estudio de sus recursos vegetales; al M.C. Enrique V. Sánchez Rodríguez, por la elaboración de la ilustración y a Clotilde Rodríguez Guerrero por la edición de la misma. El Consejo Nacional de Ciencia y Tecnología y el Consejo Estatal de Ciencia y Tecnología del Estado de Jalisco apoyaron el proyecto "inventarios florísticos y estudios estructurales y de diversidad de la vegetación de la costa sur 
de Jalisco y el estado de Colima". Los revisores anónimos dieron muy buenas sugerencias que permitieron mejorar la redacción y contenido del documento.

\section{LITERATURA CITADA}

Méndez-Larios, I. y J. L. Villaseñor. 2001. La familia Scrophulariaceae en México: diversidad y distribución. Bol. Soc. Bot. México 69: 101-121.

Molau, U. 1981. The genus Calceolaria in NW South America VI. The sections Urticopsis, Lobatae and Micranthera. Nord. J. Bot. 4: 165-185.

Molau, U. 1988. Scrophulariaceae. Part. 1. Calceolarieae. Fl. Neotrop. Monogr. 47: 1-326. 